\title{
Establishment and characterization of esophageal squamous cell carcinoma patient-derived xenograft mouse models for preclinical drug discovery
}

\author{
Jingchuan Zhang ${ }^{1,4}$, Dongxian Jiang ${ }^{2,4}$, Xiaojing $\mathrm{Li}^{2}$, Jing Lv${ }^{1}$, Liang Xie ${ }^{1}$, Li Zheng ${ }^{1}$, Paul R Gavine ${ }^{1}$, Qin $\mathrm{Hu}^{2}$,

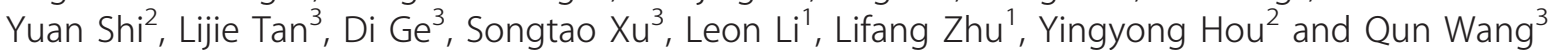

The purpose of this study was to establish and characterize patient-derived esophageal squamous cell carcinoma xenograft (PDECX) mice for utilization in antitumor drug discovery. A total of 96 esophageal squamous cell carcinoma (ESCC) tissues from Chinese patients were transplanted subcutaneously into immunodeficient mice. Histology, EGFR, K-ras, B-raf, and PIK3CA mutations, and HER2 gene amplifications were analyzed in both patient tumors and mouse xenograft tissues using immunohistochemistry, mutant-enriched liquid chip sequencing and fluorescence in situ hybridization assays, respectively. Furthermore, in vivo efficacy studies using five PDECX mice harboring a variety of genetic aberrations were performed using the chemotherapy agents 5 -fluorouracil (5-FU) and cisplatin. Thirty-seven PDECX mouse models were successfully established in immunodeficient mice. Pathological analysis revealed similar histological architecture and degrees of differentiation between patient ESCC and xenografted tumors. No mutations were identified in EGFR, K-ras, and B-raf genes in either xenograft models or patient ESCC tissues. In contrast, PIK3CA gene mutations were detected in 12.5\% (12/96) ESCC patients and 18.9\% (7/37) PDECX models. Interestingly, patient ESCC tissues exhibiting HER2 overexpression or gene amplification were unable to survive in immunodeficient mice. Further analysis showed that PDECX models carrying HER2 $2+$ expression had no response to 5-FU/cisplatin, compared with HER2-negative models. In conclusion, a panel of PDECX mouse models, which include PIK3CA mutant and HER2-positive models, was established and characterized thus mimicking the current clinical genetic setting of esophageal carcinoma. The sensitivity of HER2-negative ESCC models to chemotherapy supports stratification approaches in the treatment of esophageal carcinoma patients and warrants further investigation of the impact of PI3KCA on treatment response.

Laboratory Investigation (2014) 94, 917-926; doi:10.1038/labinvest.2014.77; published online 7 July 2014

Esophageal squamous cell carcinoma (ESCC) is one of the most prevalent cancers worldwide and occurs at a relatively high frequency ( $>50$ per 100000 population) in some areas of China. ${ }^{1,2}$ Furthermore, it has been suggested that ethnic factors can contribute to esophageal cancer susceptibility. ${ }^{1}$ ESCC is a complex disease, the mortality rate associated with ESCC is similar to the incidence rate due to diagnosis at a generally advanced stage, its aggressive characteristics, and a paucity of effective treatment strategies. ${ }^{3}$ In spite of its poor prognosis, however, ESCC has not been well studied to date.

Surgery remains one of the fundamental treatment strategies, however, the 5-year survival for nonmetastatic disease is $20-40 \% .{ }^{4}$ Patients with metastatic disease who are treated with palliative chemotherapy using a combination of 5-fluorouracil (5-FU) and cisplatin have a median survival of less than 1 year with response rates of $25-45 \%$ in phase II and III trials. ${ }^{3,5}$ Salvage options for patients with refractory ESCC are rare. ${ }^{6}$ Recently, neoadjuvant treatment approaches using a combination of chemotherapy and radiotherapy have been performed on locally advanced EC, with some improvements observed in clinical trials. ${ }^{7-9}$ Despite these limited improvements, the prognosis for these patients remains poor. ${ }^{10}$ In addition, the trimodal therapeutic model (use of cetuximab in combination with definitive

\footnotetext{
${ }^{1}$ Innovation Center China, AstraZeneca Global R\&D, Zhangjiang Hi-Tech Park, Shanghai, People's Republic of China; ${ }^{2}$ Department of Pathology, Zhongshan Hospital, Fudan University, Shanghai, People's Republic of China and ${ }^{3}$ Department of Thorax Surgery, Zhongshan Hospital, Fudan University, Shanghai, People's Republic of China

Correspondence: Dr Y Hou, MD, Department of Pathology, Zhongshan Hospital, Fudan University, Shanghai 200032, People's Republic of China or Dr Q Wang, MD, Department of Thorax Surgery, Zhongshan Hospital, Fudan University, Shanghai 200032, People's Republic of China.

${ }^{4}$ These authors contributed equally to this work.

Received 15 February 2014; revised 27 March 2014; accepted 8 April 2014
} 
chemoradiotherapy) appeared to offer hope in a phase IB/II clinical trial, ${ }^{11}$ but failed in a later phase IIB/III clinical trial. ${ }^{12}$ Furthermore, the efficacy of new therapeutic drugs remains to be tested in ESCC as most of the previous trials have included predominantly esophageal adenocarcinoma patients. ${ }^{13-19}$

Despite some successes in the treatment of cancers, ${ }^{9}$ more novel rational therapies targeting specific cancer-related molecules are urgently needed. The increasing body of knowledge around molecular and genetic events involved in oncogenesis and tumor progression has led to the identification of new therapeutic targets and the synthesis of novel therapeutic agents. Animal models have been used in front-line preclinical studies for predicting the efficacy and possible toxicities of anticancer drugs in cancer patients. ${ }^{20}$ Although the advent of cancer cell-line culture techniques fuelled an acceleration and expansion of cancer biology discovery that continues to this day, the harsh reality is that there is a high failure rate among new oncology agents when attempting to translate preclinical efficacy into clinical benefit. One of the most frequently cited reasons is the lack of preclinical models that recapitulate the heterogeneity of tumors in patients. ${ }^{21,22}$

Human tumor fragments obtained from patients and directly transplanted into immunodeficient mice, known as primary xenografts or tumor grafts, are one category of recognized models used as tools for preclinical assays. ${ }^{23-28}$ Although the methods have been established for decades, these preclinical models are only now being consistently characterized and applied to drug development in oncology. There is a strong evidence that a greater genetic divergence exists between a primary tumor and the corresponding cell line derived from that tumor, versus a direct xenograft. ${ }^{22,29}$

Future preclinical evaluation of these and other agents for ESCC requires the development of animal models of human ESCC. To obtain preclinical results with high predictive value for clinical trials, the choice of the tumor models on which new compounds and novel drug combinations are evaluated is critical.

In the present study, we used fresh patient ESCC tissues to establish patient-derived esophageal squamous cell carcinoma xenograft (PDECX) models in immunodeficient mice to support drug discovery and development.

\section{MATERIALS AND METHODS Patients and Tissue Samples}

From March to August 2010 with the assistance of the Department of Thorax Surgery, Zhongshan Hospital, 96 patient primary ESCC tissues without any preoperative treatment were obtained intraoperatively during esophagetomy resection at Zhongshan Hospital. None of the patients received any therapies before surgery. Prior written informed consent was provided from all patients and the study protocol received Ethics Board approval at Zhongshan
Hospital, Fudan University. Where sufficient residual tumor tissue was available, histopathological examination and related analyses were performed. The tumor specimens were collected at surgery and divided into three parts for the following purposes: implantation into immunodeficient mice for xenograft model establishment, snap freezing in liquid nitrogen for DNA/RNA extraction, and fixed in 10\% formalin buffer for $24 \mathrm{~h}$ and embedded into paraffin for immunohistochemical (IHC) analysis.

\section{Clinical Data Collection}

The diagnosis of ESCC was confirmed by histological analysis in all cases. Then, the following patient characteristics were collected for research study only, including: gender, age, tumor site (upper, middle, and lower of esophagus), histological grade, coagulative necrosis, nerve and vascular infiltration, mitotic index (number recorded as $<50 / 10 \mathrm{HPF}$, or $\geq 50 / 10 \mathrm{HPF}$ ), lymph node metastasis, and stage as previously reported. ${ }^{4}$

\section{Establishment of PDECX Mouse Models}

Eight- to ten-week-old female nude $(n u / n u)$ and the severe combined immune-deficient (SCID) mice (Vital River, Beijing, China) were used for xenograft model generation in this study. All experiments with immunodeficient mice were performed in accordance with the guidelines approved by IACUC. The PDECX models were established with fresh patient ESCC tissues, surgically removed from ESCC patients, as described in a previous study. ${ }^{30}$ In brief, surgically removed patient's ESCC tissues (F0 tissue) were cut into fragments of approximately $15 \mathrm{~mm}^{3}$ in volume and implanted subcutaneously via Trocar needle into female SCID mice within $2 \mathrm{~h}$ after surgery. Patient tumor-implanted mice were observed daily until 90 days. Tumors were measured once a week by caliper when tumors started to grow up in mice. The $\sim 500 \mathrm{~mm}^{3}$ xenografted ESCC tumors subsequently harvested from the mice were further implanted and expanded in nude mice. After three consecutive mouseto-mouse passages, the xenograft was considered to be stable and was characterized. The xenografted tumor specimens in each passage of tumor-bearing mice were harvested and divided into three parts for the following purposes. The first was implanted into immunodeficient mice for the next generation of the xenograft model. The second part was used for DNA/RNA extraction by snap freezing in liquid nitrogen. The third part was fixed in $10 \%$ formalin buffer for $24 \mathrm{~h}$ and embedded into paraffin for IHC analysis. Fresh tumor fragments at passages 3-5 were frozen with $20 \%$ FCS in liquid nitrogen for further model recovery and utilization for drug discovery.

\section{H\&E and IHC Staining}

Tissues from all PDECX models and the corresponding ESCC patient tumors were harvested and fixed in 10\% buffered formalin within $30 \mathrm{~min}$ after resection according to our 
previous study. Sections were stained with hematoxylin and eosin and reviewed by two pathologists to confirm the ESCC diagnosis. Dual cores from each tissue were made on tissue microarray (TMA) for IHC and fluorescence in situ hybridization analysis. For the IHC, all incubations were at room temperature and washes were performed with TBST. Antigen retrieval was performed in a pressure cooker at $110^{\circ} \mathrm{C}$ for $5 \mathrm{~min}$ in retrieval buffer (S2367, DAKO). Endogenous peroxidase activity was blocked with $3 \%$ hydrogen peroxide (S2023, DAKO). Sections were then incubated with anti-HER2 antibody (HerceptestTM, DAKO) for $30 \mathrm{~min}$. Immunocomplexes were detected by incubation with DAKO K5204 for $30 \mathrm{~min}$ and were visualized with diaminobenzidine (K3468, DAKO) for $10 \mathrm{~min}$. For HER2-negative cases, the experiment was repeated on the whole section in order to exclude heterogeneity. The IHC scoring criteria on ESCC followed Hoffman's criteria on human gastric cancer: ${ }^{31}$ no staining or $<10 \%$ tumor cell-positive staining as 0/negative; faintly or barely perceptible staining on $>10 \%$ tumor cell membrane as $1+$; weak to moderate positive staining on $>10 \%$ tumor cells as $2+$; as tumor tissues on TMA mimic the biopsy samples, cohesive moderate to strong staining on the membranes were scored as $3+$.

\section{HER2 Fluorescence In Situ Hybridization Assay}

TMA sections were dewaxed and dehydrated. Tissue pretreatment was performed according to the SpotLight Tissue pretreatment kit manual (Invitrogen, Cat \#00-8401). After pretreatment, $10 \mu \mathrm{l}$ HER2/CEP17 probes were applied onto each TMA slide, covered with coverslip and circled around the edge of coverslip with rubber cement (Mpbio, Cat \#11FIXO0125). Sections together with probes were codenaturated at $75{ }^{\circ} \mathrm{C}$ for $4 \mathrm{~min}$ on OmniSlide In-Situ hybridization System, then incubated at $37^{\circ} \mathrm{C}$ overnight. After codenaturation and incubation, probes were washed off sequentially with $0.3 \% \mathrm{NP} 40 / 1 \times \mathrm{SSC}$ at $75.5^{\circ} \mathrm{C}$ for $3 \mathrm{~min}$, and $0.3 \% \mathrm{NP} 40 / 2 \times \mathrm{SSC}$ at $75.5^{\circ} \mathrm{C}$ for $2 \mathrm{~min}, 2 \times \mathrm{SSC}$ at room temperature $2 \mathrm{~min}$, two times, then dehydrated sequentially in 70,85 and $100 \%$ ethanol, and air dried. Sections were mounted with $0.3 \mu \mathrm{g} / \mathrm{ml}$ DAPI mounting medium (diluted from Vector, Cat \#H-1200), and stored at $4{ }^{\circ} \mathrm{C}$ to avoid light for at least $30 \mathrm{~min}$ before scoring.

In each case, 50 tumor nuclei were evaluated. Cases showing average gene copy number of HER2 $>6$ or a gene/CEN17 fluorescence ratio $\geq 2$ were considered gene amplified. Amplification was also defined in cases where HER2 signals formed a tight gene cluster. The above criteria are based on Hoffmann's criteria ${ }^{31}$ and related investigations. ${ }^{32,33}$

\section{Gene Mutation Screening}

Formalin-fixed paraffin-embedded tumor blocks were reviewed for quality and tumor content. A single representative block, from either the patient primary tumor or the xenograft model, containing at least $70 \%$ of neoplastic cells, was selected for gene analysis. Genomic DNA was extracted using the QIAamp Mini kit (Qiagen) according to the manufacturer's instructions. Based on the prevalence of gene mutations and molecular segmentation in other tumor types (as well as our previously published data; Hou et al), ${ }^{34}$ 'hot spot' EGFR, K-ras, B-raf, and PIK3CA gene mutations were screened for using the mutant-enriched PCR method. ${ }^{35}$ The method was performed by SurExam Bio-Tech Co. Ltd., Guangzhou Technology Innovation Base, Science City, Guangzhou, PRC. Positive results were further confirmed by PCR-direct sequencing. The primers for exon 9 of PIK3CA gene were $5^{\prime}$-GTCTTAGATTGGTTCTTTCCTG- $3^{\prime}$ for forward and $5^{\prime}$-GCATTTAATGTGCCAACTACC- $3^{\prime}$ for reverse, for exon 20 of PIK3CA gene were $5^{\prime}$-TTTGTCTACGA AAGCCTCTCTA- $3^{\prime}$ for forward and $5^{\prime}$-CCATCACTTTTT CCTTCTCCAT- $3^{\prime}$ for reverse.

\section{Antitumor Efficacy of Cytotoxic Agents}

For in vivo efficacy studies, xenograft tumor-bearing nude mice (tumor volumes of $150-250 \mathrm{~mm}^{3}$ ) were sorted randomly and assigned to treatment groups (eight animals per group) or the vehicle control. The treatment groups received the chemotherapy drugs $5-\mathrm{FU} / \mathrm{cisplatin}(5-\mathrm{FU}$ at $10 \mathrm{mg} / \mathrm{kg} / \mathrm{qd}$ for 5 days a week intraperitoneally, and cisplatin at $5 \mathrm{mg} / \mathrm{kg} /$ qd/once a week on the first day of the week, intravenously). Subcutaneous xenografted tumors in nude mice and mice body weight were measured twice a week. Tumor volumes were calculated by measuring two perpendicular diameters with calipers. Tumor volumes $(V)$ were calculated by using the formula: $V=\left(\right.$ length $\left.\times[\text { width }]^{2}\right) / 2$. Percentage tumor growth inhibition (\%TGI) was calculated using the formula: \%TGI $=[1-($ change of tumor volume in treatment group/change of tumor volume in control group) $] \times 100$ and was used for the evaluation of antitumor efficacy. Four stratification groups were defined based on criteria in previous publications: ${ }^{24}$ (a) high responders, in which the treatment induced complete regressions (the established tumors being no longer palpable after treatment); (b) moderate responders, in which TGI was superior to 50\%; (c) low responders, with TGI between 40 and 50\%; and (d) the non-responders, in which the growth parameters were not significantly altered by the treatment.

\section{Statistical Analysis}

A $\chi^{2}$ test was used for univariate analyses, cross-tabulations with qualitative variables were analyzed with the Fisher's exact, with a $P$ value less than 0.05 recorded as significant in clinical data analysis. To evaluate statistical significance in our antitumor efficacy studies, the Student's $t$-test was used to compare TGI in treatment groups to the control group. Statistical tests were two sided, with $P<0.05$ considered significant. 


\section{RESULTS \\ Establishment of Patient-Derived ESCC Xenograft Mouse Models}

A total of 96 ESCC samples obtained from esophageal cancer patients were implanted subcutaneously into SCID mice, and growing xenograft tissues then implanted into nude mice starting from the second generation of xenograft model. Thirty-seven PDECX mouse models were established in consecutive passages in nude mice (Figure 1). The PDECX model success rate was $53.1 \%(51 / 96)$ in the first generation in SCID mice, and 92.2\% (47/51), 89.4\% (42/47), 88.1\% (37/ 42) from second to fourth generations in nude mice, respectively. After the fifth generation in nude mice, PDECX mouse models became stable without further change in model survival. The overall survival rate of the PDECX models during model establishment was 38.5\% (37/96; defined as the generation of a stable PDECX model in an immunodeficient mouse from initial implantation of primary patient ESCC tissue). All 37 established PDECX mouse models showed kinetic growth (data not shown), similar to

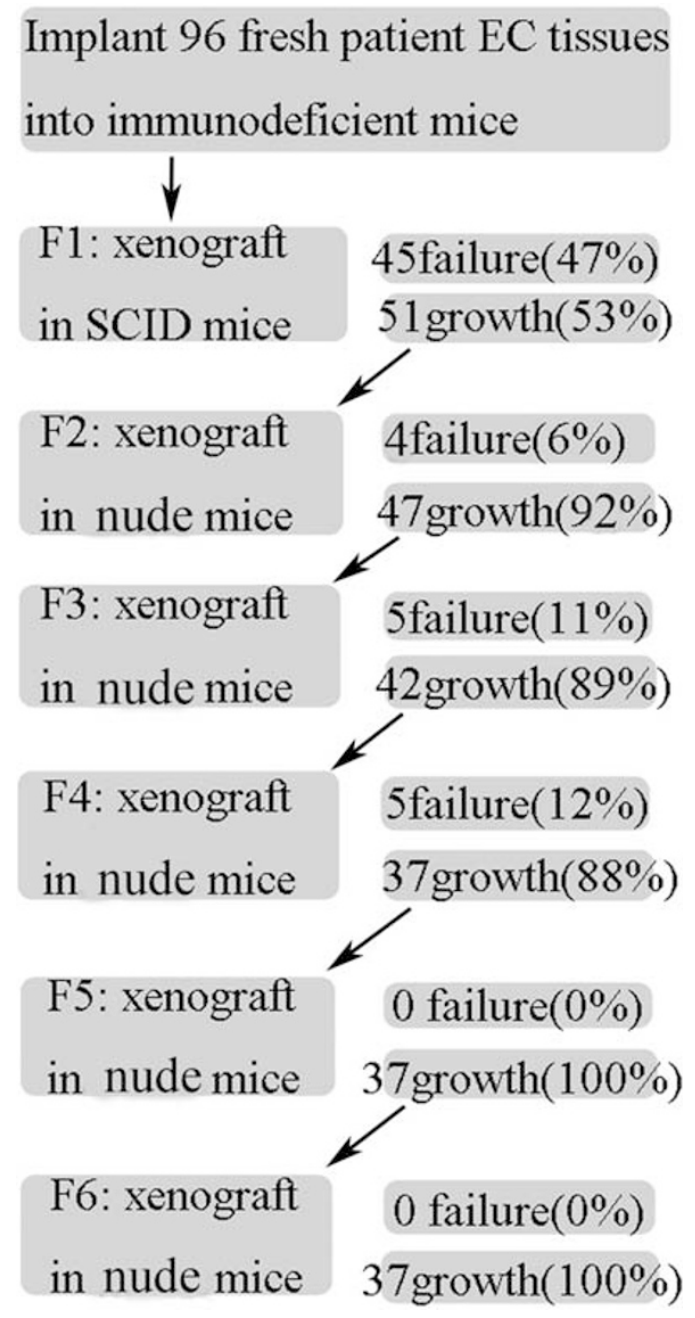

Figure 1 Success rates of PDECX model development at different passages. the growth patterns obtained in our previous studies. ${ }^{30}$ Examination of autopsies in ESCC-bearing mice from these xenograft mouse models at 2-3 months post-implantation revealed no evidence of metastases in the brain, lung, liver, or kidney. All established PDECX models could be recovered in mice after freeze/thawing of xenograft tumor fragments using liquid nitrogen (data not shown). Pathology assessment by $\mathrm{H} \& \mathrm{E}$ staining and analysis by pathologist revealed similar morphologies in PDECX tissues compared with that of the corresponding patient primary ESCC tissues in most cases (Figure 2a).

\section{Analysis of Potential Factors Affecting PDECX Model Establishment Success}

Results of our correlation analyses between the success rates of PDECX model establishment and patient clinical parameters are listed in Table 1. Of all analyzed parameters, nerve invasion, necrosis, and HER2 amplification or over-expression in patient ESCC tissues showed correlative trends, but unfortunately did not reach statistical significance. $(P=0.088,0.118,0.106$, respectively; Table 1$)$. In 96 patient esophageal carcinoma tissues, 3 cases showed HER2 $3+$ expression by IHC analysis and 1 case showed HER2 gene amplification. A total of 4.2\% (4/96) cases were evaluated as HER2 gene amplified or overexpressing. Surprisingly, none of the ESCC tissues showing HER2 amplification or IHC $3+$ expression were able to grow up in immunodeficient mice. Compared with HER2 as a potential negative factor, PIK3CA mutation represents an unlikely factor in the successful establishment of PDECX mouse models (Table 1). A total of $41.6 \%(5 / 12)$ of patient ESCC tissues harboring PIK3CA mutation grew up in immunodeficient mice (Table 1).

\section{Characterization of PDECX Mouse Models}

In order to characterize our established PDECX mouse models at both gene and protein levels, we screened for the major known genetic aberrations in esophageal carcinoma, both in model tissues and patient tumors, and performed IHC analysis. The list included mutations in PIK3CA, EGFR, $K$-ras, B-raf, and expression of HER2 protein in our 37 established PDECX models and the corresponding patient ESCC tissues.

HER2 positivity (IHC score $1+, 2+$ or $3+$ ) was identified in $24.3 \%(9 / 37)$ of patient ESCC samples and $32.4 \%$ (12/37) of PDECX models. A total of 76\% (28/37) of PDECX models showed consistent HER2 expression (positive or negative) compared with the corresponding patient ESCC tissues (Table 2 and Figure $2 \mathrm{~b}$ ).

Gene mutations within EGFR (exons 18, 19, 20, 21), K-ras (exons 2 and 3), B-raf (exons 11 and 15), and PIK3CA (exons 9 and 20) were screened for within all 37 established PDECX model and patient ESCC tissues. Interestingly, no mutations were detected in EGFR, K-ras, or B-raf in any of the samples. PIK3CA mutation, however, was identified in $13.5 \%(5 / 37)$ of patient ESCC tissues (Figure 3) and 18.9\% (7/37) of PDECX 
a

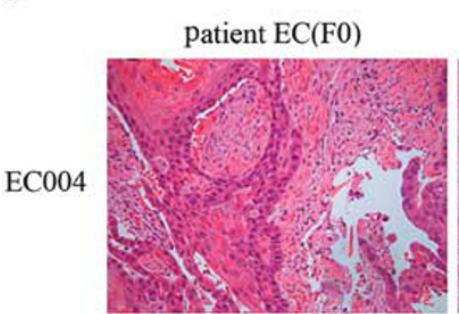

G2

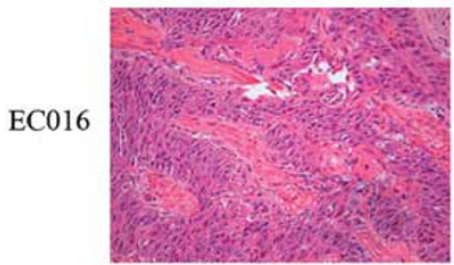

G3

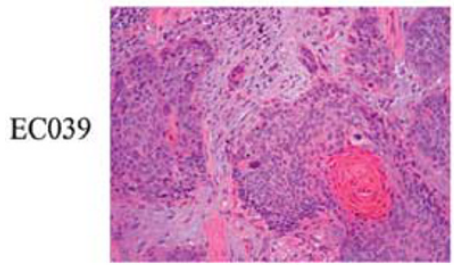

G2

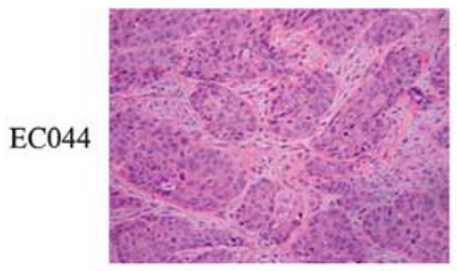

G3

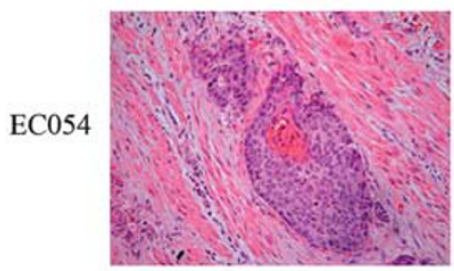

G2
HE staining

xenograft EC(F3)

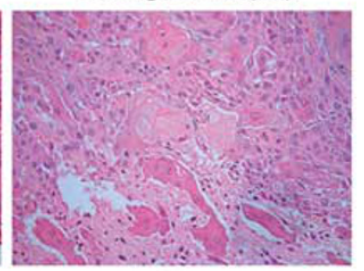

G2

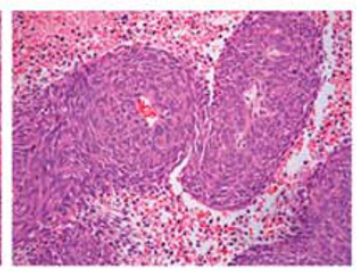

G3

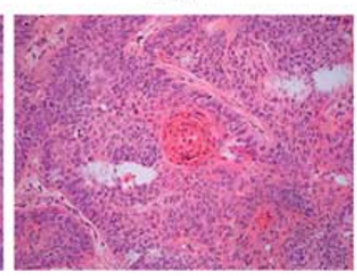

G2

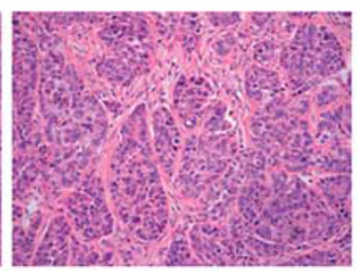

G3

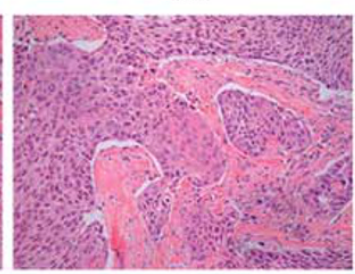

G3 b

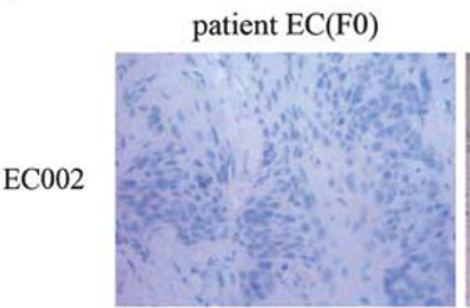

0

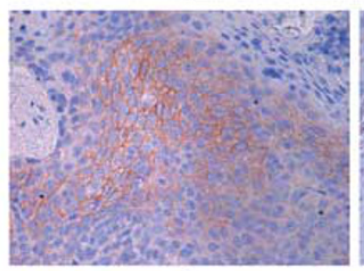

$2+$

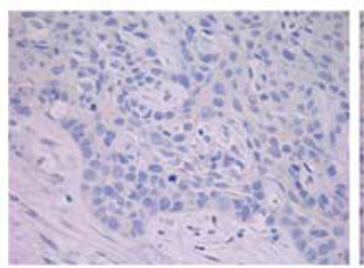

0

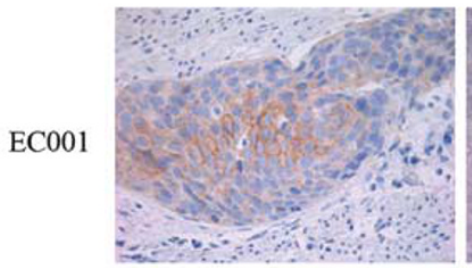

$2+$

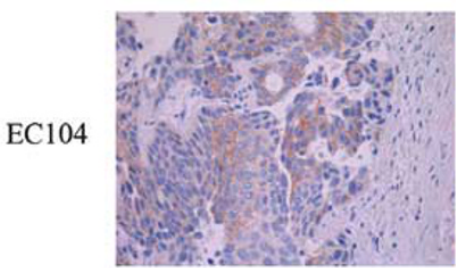

$2+$
HER2 IHC

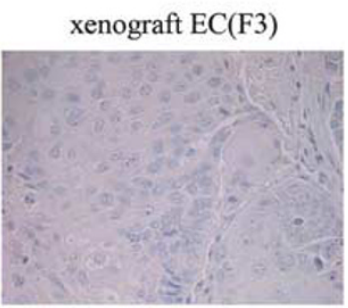

0

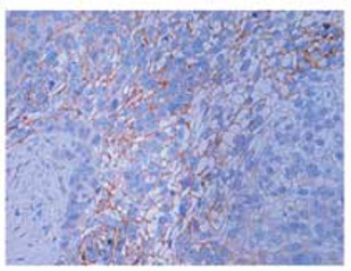

$2+$

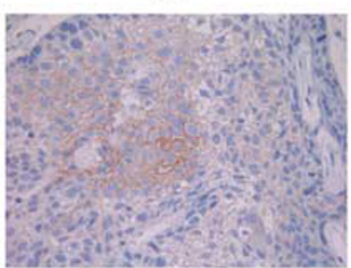

$2+$

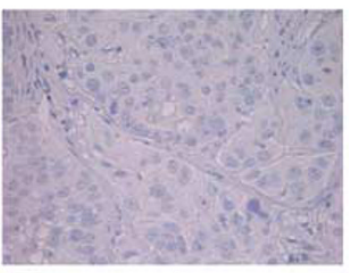

0

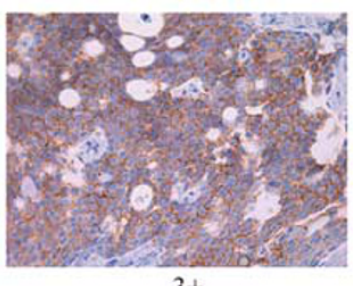

$3+$

Figure 2 Comparison of pathology and HER2 expression between patient ESCC tissues and corresponding PDECX models. In most cases, tumors show similar architecture, differentiation, and HER2 expression. Some variations in HER2 expression were observed in EC047, EC001, and EC104.

models. A total of 71\% (5/7) of PDECX models showed consistent PIK3CA mutation when compared with the corresponding patient ESCC tissues. Interestingly, PIK3CA mutation in two additional PDECX models was not detected in the corresponding patient ESCC tissues.

\section{PDECX Chemotherapy Anti-Tumor Efficacy Studies}

To evaluate whether our PDECX mouse models responded to treatment with standard ESCC antitumor drugs, five available PDECX models were treated with the clinically used cytotoxic agents, 5-FU and cisplatin (Figure 4). Our data demonstrated that two PDECX models with genetic aberrations (HER2 IHC $2+$ in EC044; HER2 IHC $2+$ and PI3KCA mutation in EC039) had no significant response to cytotoxic agents $(P>0.05)$. Interestingly, however, three PDECX mouse models with HER2 negativity and wild-type PIK3CA were sensitive to 5-FU/cisplatin (49\% TGI in EC004, $P<0.05$; $47 \%$ TGI in EC016, $P<0.05$; and $84 \%$ TGI in EC054, $P<0.001)$. EC004 and EC016 were categorized as low responders, whereas EC054 was a moderate responder based on predefined response criteria. $^{24}$ 
Table 1 Clinical features of ESCC patients and in vivo tumor take rate (\%) by univariate analysis based on 96 cases

\begin{tabular}{|c|c|c|c|}
\hline $\begin{array}{l}\text { Clinicopathological } \\
\text { features }\end{array}$ & $\begin{array}{c}\text { Patient } \\
\text { number }(n)\end{array}$ & $\begin{array}{l}\text { Successful xenograft } \\
\text { (tumor take rate) }\end{array}$ & $P$ value \\
\hline Sex & & & 0.639 \\
\hline Male & 80 & $30(38 \%)$ & \\
\hline Female & 16 & 7 (44\%) & \\
\hline Age at diagnosis (years) & & & 0.824 \\
\hline$<60$ & 35 & $14(40 \%)$ & \\
\hline$\geq 60$ & 61 & $23(38 \%)$ & \\
\hline Tumor site & & & 0.628 \\
\hline Upper + middle & 34 & $12(35 \%)$ & \\
\hline Lower & 62 & $25(40 \%)$ & \\
\hline Differentiation & & & 0.831 \\
\hline $\mathrm{G} 2$ & 61 & $24(39 \%)$ & \\
\hline G3 & 35 & $13(37 \%)$ & \\
\hline Nerve invasion & & & 0.088 \\
\hline Yes & 34 & $17(50 \%)$ & \\
\hline No & 62 & $20(32 \%)$ & \\
\hline Vascular invasion & & & 0.722 \\
\hline Yes & 19 & $8(42 \%)$ & \\
\hline No & 77 & 29 (38\%) & \\
\hline Necrosis & & & 0.118 \\
\hline Yes & 58 & $26(45 \%)$ & \\
\hline No & 38 & $11(29 \%)$ & \\
\hline Lymph node metastases & & & 0.661 \\
\hline Yes & 44 & $18(41 \%)$ & \\
\hline No & 52 & $19(37 \%)$ & \\
\hline Mitoses (10 HPF) & & & 0.843 \\
\hline$<50$ & 66 & $25(38 \%)$ & \\
\hline$\geq 50$ & 30 & $12(40 \%)$ & \\
\hline Stage & & & 0.163 \\
\hline । & 4 & $0(0 \%)$ & \\
\hline$\|$ & 53 & $19(36 \%)$ & \\
\hline III & 39 & $18(46 \%)$ & \\
\hline PIK3CA mutation & & & 0.812 \\
\hline Yes & 12 & $5(42 \%)$ & \\
\hline No & 84 & $32(38 \%)$ & \\
\hline HER2 amplification or $3+$ & & & 0.106 \\
\hline Yes & 4 & $0(0 \%)$ & \\
\hline No & 92 & 37 (40\%) & \\
\hline
\end{tabular}

\section{DISCUSSION}

For several decades, historic cancer drug screening efforts have relied heavily on the use of mouse tumor xenograft models. A key problem, however, is that the source of these xenografts are permanent cell lines kept under continuous
Table 2 Comparison between patient ESCC tissue and corresponding patient-derived ESCC xenograft tissue

ID Patient ESCC tissue Patient-derived ESCC xenograft tissue

HER2 IHC EXON 9 of PIK3CA HER2 IHC

Exon 9 of PIK3CA

\begin{tabular}{|c|c|c|c|c|}
\hline EC001 & $2+$ & Wt & - & Wt \\
\hline EC002 & - & Wt & - & Wt \\
\hline EC004 & - & Wt & - & $W t$ \\
\hline EC006 & - & Wt & - & Wt \\
\hline EC008 & $2+$ & $W t$ & - & Wt \\
\hline EC014 & $2+$ & Wt & $1+$ & Wt \\
\hline EC016 & - & $W t$ & - & $W t$ \\
\hline EC018 & - & Wt & - & Wt \\
\hline EC019 & - & Wt & - & Wt \\
\hline EC021 & - & Wt & - & Wt \\
\hline EC022 & - & $W t$ & $1+$ & Wt \\
\hline EC023 & $1+$ & Wt & $1+$ & Wt \\
\hline EC030 & - & $\mathrm{GAG}>\mathrm{AAG}$ & $1+$ & $\mathrm{GAG}>\mathrm{AAG}$ \\
\hline EC039 & $2+$ & $\mathrm{GAA}>\mathrm{AAA}$ & $2+$ & $\mathrm{GAA}>\mathrm{AAA}$ \\
\hline EC040 & - & $W t$ & - & Wt \\
\hline EC043 & $2+$ & Wt & $1+$ & Wt \\
\hline EC044 & $2+$ & Wt & $2+$ & Wt \\
\hline EC047 & - & Wt & $2+$ & Wt \\
\hline EC054 & - & Wt & - & Wt \\
\hline EC056 & - & Wt & - & Wt \\
\hline EC057 & - & Wt & $2+$ & Wt \\
\hline
\end{tabular}

GAG $>$ AAG - $\quad$ GAG $>$ AAG

$\begin{array}{lllll}\mathrm{ECO} & - & \mathrm{GAG} & >\mathrm{AAG} & -\end{array}$

EC063 - $\quad$ Wt $\quad$ W

$\begin{array}{lllll}\mathrm{ECO} 06 & - & \mathrm{Wt} & \mathrm{W} & \mathrm{Wt}\end{array}$

$\begin{array}{llllll}\mathrm{ECO} & - & \mathrm{Wt} & \mathrm{W} & \mathrm{Wt}\end{array}$

ECO74 - $\quad$ GAG $>$ AAG $\quad-\quad$ GAG $>$ AAG

$\begin{array}{lllll}\mathrm{ECO} 77 & - & \mathrm{Wt} & - & \mathrm{Wt}\end{array}$

$\begin{array}{lllll}\mathrm{ECO} & - & \mathrm{Wt} & - & \mathrm{Wt}\end{array}$

$\begin{array}{llllll}\mathrm{ECO} & - & & \mathrm{Wt} & \mathrm{W} & \mathrm{Wt}\end{array}$

$\begin{array}{llllll}\mathrm{ECO} 83 & - & \mathrm{Wt} & 1+ & \mathrm{Wt}\end{array}$

$\begin{array}{llllll}\mathrm{ECO} 87 & - & \mathrm{Wt} & - & \mathrm{Wt}\end{array}$

$\begin{array}{lllll}\text { ECO89 } & - & \text { Wt } & 2+ & \text { Wt }\end{array}$

$\begin{array}{lllllll}\mathrm{ECO} 2 & - & \mathrm{Wt} & \mathrm{W} & \mathrm{Wt}\end{array}$

$\begin{array}{llllll}\mathrm{ECO} & - & \mathrm{Wt} & - & \mathrm{Wt}\end{array}$

$\begin{array}{lllll}\mathrm{EC} 104 & 2+ & \text { Wt } & 3+ & \mathrm{GAA}>\mathrm{AAA}\end{array}$

$\begin{array}{lllll}\text { EC105 } & 1+ & \text { Wt } & - & \text { Wt }\end{array}$

$\mathrm{Wt}$, wild type.

in vitro culture conditions, resulting in xenograft tumors, which are mostly undifferentiated and bearing weak resemblance to the real human disease histology and tumor 


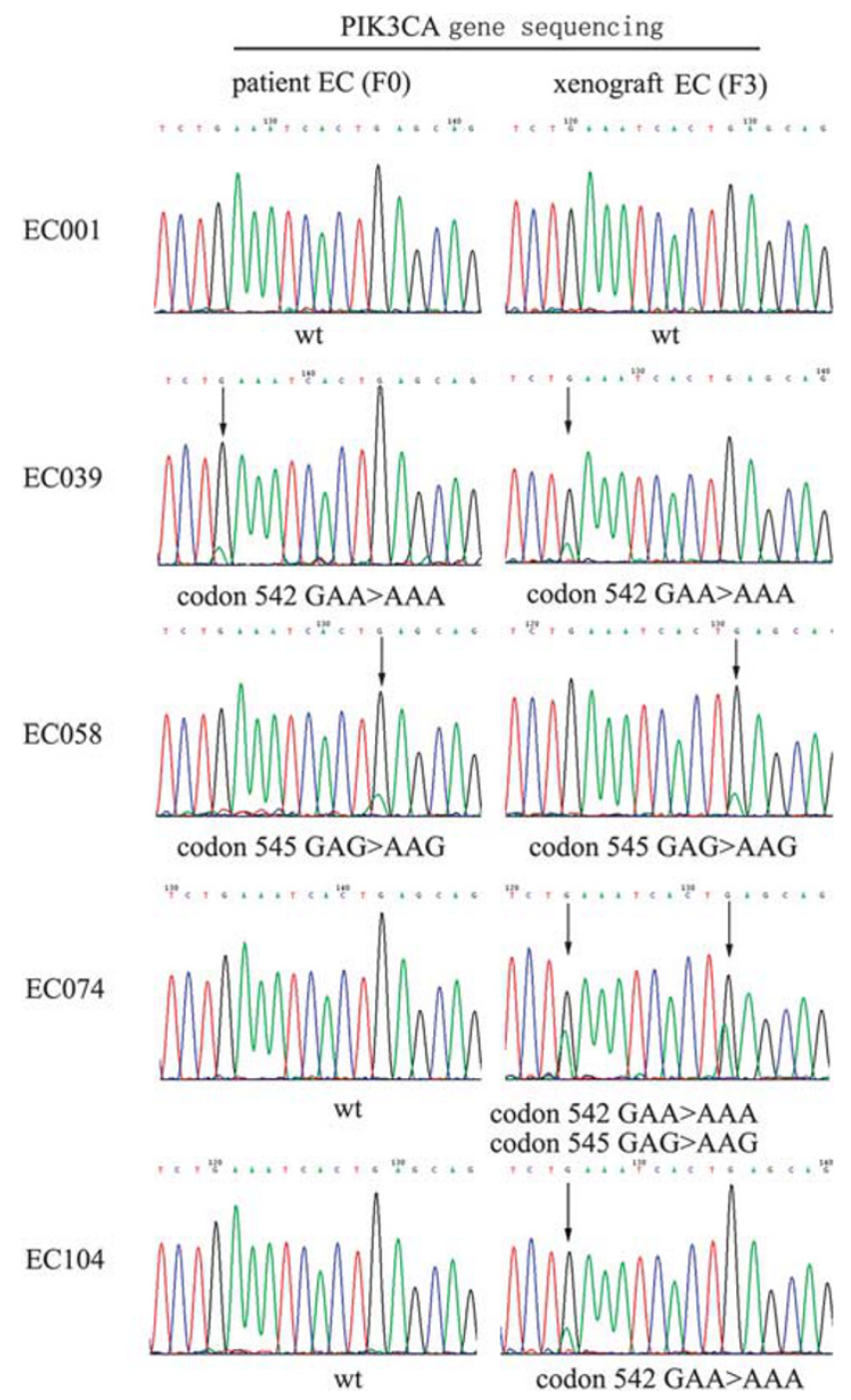

Figure 3 PIK3CA mutation in patient ESCC and the corresponding PDECX models. EC001 harbors a wild-type PIK3CA gene in primary tissue and xenografted model, EC039 contains a codon 542 GAA > AAA mutation, and EC058 has a codon 545 GAG > AAG mutation in exon 9 of the PIK3CA gene in both primary and xenografted tissues, EC074 has acquired a codon 542 GAA > AAA mutation as well as a codon 545 GAG $>$ AAG mutation in xenografted tissue, EC104 acquired a codon 542 GAA $>$ AAA mutation in xenografted tissue.

architecture. ${ }^{25,36}$ Development of esophageal animal xenograft models has been especially difficult but some success has been achieved, ${ }^{37}$ either by the inoculation of EC cell lines into immunodeficient rodents (mice and rats) or rabbits. ${ }^{15,38-41}$ Unfortunately, again, however, these cell lines also suffer from the same issues of long in vitro culture times and loss of original tumor characteristics. Another problem faced by the field of esophageal oncology research is that most of the commercially available EC cell lines were originally derived from esophageal adenocarcinoma, ${ }^{15,39,40,42}$ which does not represent the predominant ESCC disease in Asian esophageal cancer patients. Furthermore, our recent experimental results show that some of the commercially available EC cell lines have lost their original genetic aberrations, such as PIK3CA mutation, in the early generations. Therefore, the establishment of clinically relevant animal xenograft tumor models is necessary and urgently required to progress preclinical ESCC-targeted drug discovery. In recent years, patient-derived xenograft mouse models have been established through direct transplantation of fresh patient tumor tissue into immunodeficient mice. ${ }^{23,43}$ As early as 1981, Kitamura et al ${ }^{44}$ established a panel of 17 PDECX models. Although similar models have been established in Western countries, ${ }^{45}$ the majority of PDECX models were of adenocarcinoma origin. Therefore, our present study aimed to establish a panel of Chinese patient-derived ESCC xenograft mouse models, harboring a variety of genetic aberrations and thereby constituting a pharmacologic platform for targeted drug efficacy evaluation.

In our study, a total of 37 viable and recoverable PDECX mouse models were established by implanting 96 fresh Chinese ESCC tissues into immunodeficient mice. Our successful engraftment rate of $38.5 \%$ was in the range of engraftment rates observed in similar patient-derived xenograft mouse model studies. $^{28,46-49}$ Correlative analysis between patient clinical characteristics and PDECX model establishment success rates demonstrated no statistically significant factors, but did, however, show potential negative trends associated with nerve invasion status and HER2 amplification or overexpression.

Based on published molecular analyses of the esophageal genetic landscape, together with our own published data (Hou et al, Boland and Burtness), ${ }^{34,50}$ we chose to focus on EGFR, KRas, BRaf, HER2, and PIK3CA. HER2 amplification and protein overexpression have been observed in ESCC and many other tumor types ${ }^{33,51}$ and often associates with poor prognosis. ${ }^{31,52-54}$ We identified $4.2 \%$ (5/96) of patient ESCC tissues showing overexpression or amplification of the HER2 gene, which was lower than previous reports. ${ }^{33}$ Unexpectedly, patient ESCC tissues with HER2 gene amplification or overexpression (HER2 IHC $3+$ ) were unable to form stable PDECX models in immunodeficient mice. The majority of the failures (3/5) occurred in generation 1 (F1), whereas two models survived in the first generation and then failed in the second generation. These data are consistent with published observations of breast cancer xenograft development, where the establishment of patient-derived xenograft mouse models harboring HER2 amplification is rare. ${ }^{24}$ Unfortunately, the underlying mechanism of how HER2 amplification or overexpression could contribute to model failure is currently unclear. We also acknowledge that the relatively low incidence of HER2 gene amplification or overexpression in this data set $(4.2 \%)$ could likely introduce some bias in our statistical analyses.

In contrast to the negative impact of HER2 gene amplification on successful PDECX model establishment, PIK3CA gene mutation had no apparent impact. In all, 41.6\% (5/12) of patient ESCC tissues harboring PIK3CA mutation grew up 

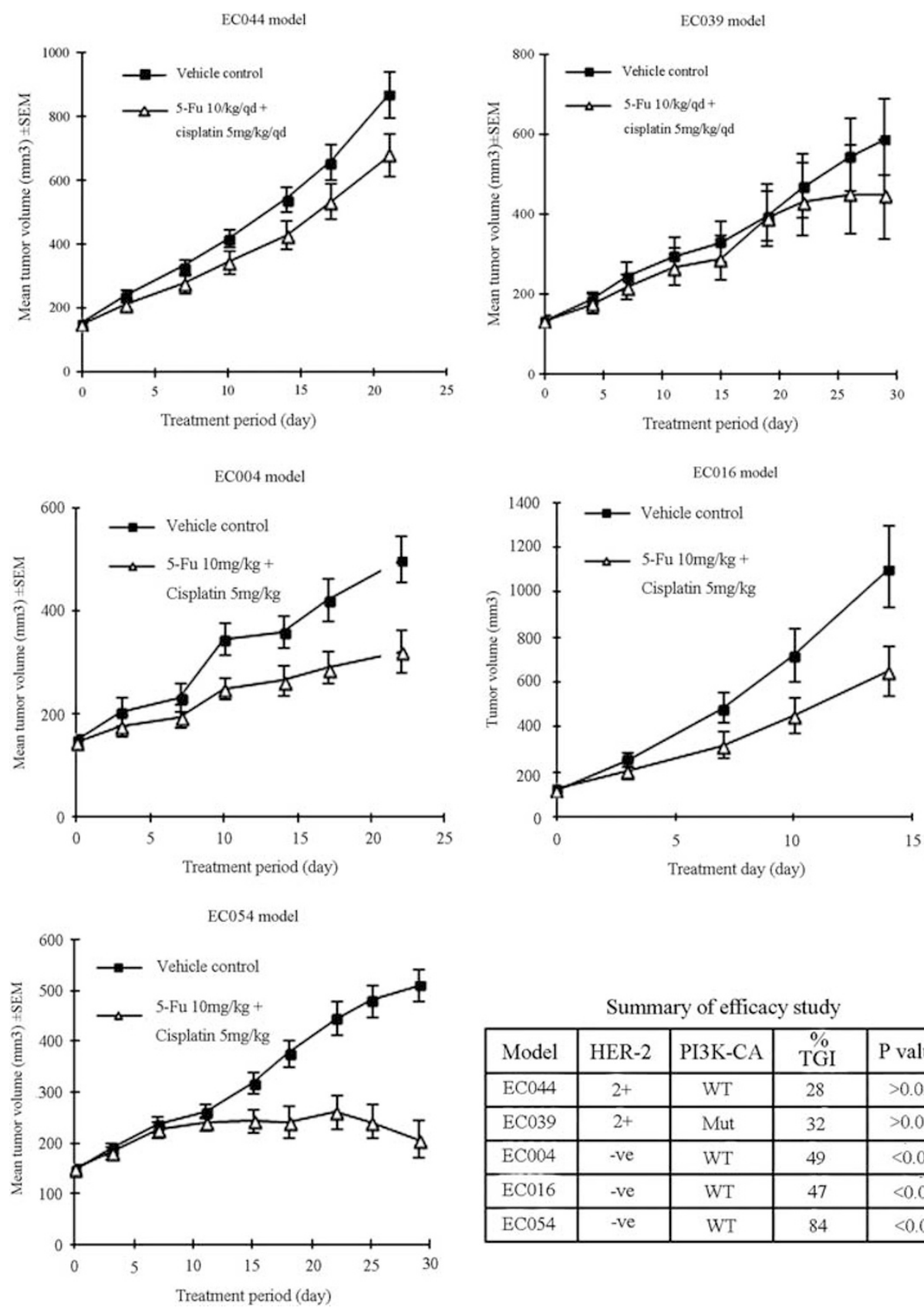

\begin{tabular}{|c|c|c|c|c|}
\multicolumn{7}{c}{ Summary of efficacy study } \\
\hline Model & HER-2 & PI3K-CA & $\begin{array}{c}\% \\
\text { TGI }\end{array}$ & P value \\
\hline EC044 & $2+$ & WT & 28 & $>0.05$ \\
\hline EC039 & $2+$ & Mut & 32 & $>0.05$ \\
\hline EC004 & -ve & WT & 49 & $<0.05$ \\
\hline EC016 & -ve & WT & 47 & $<0.05$ \\
\hline EC054 & -ve & WT & 84 & $<0.001$ \\
\hline
\end{tabular}

Figure 4 In vivo efficacy of chemotherapy in five PDECX mouse models. Tumor-bearing mice were treated with 5-FU and cisplatin starting from tumor volumes of $100-200 \mathrm{~mm}^{3}$. Subcutaneous tumor volumes were measured using calipers and calculated and expressed as mean \pm s.e.m.

in immunodeficient mice, similar to the overall success rate $(38.5 \%)$ of model establishment in this study. Interestingly, two additional PDECX models were identified as PIK3CA mutant, whereas the corresponding patient ESCC tissues were identified as PIK3CA wild type. We also observed that a further two PDECX models lost HER2 expression (from IHC $2+)$ in the corresponding patient ESCC tissues to negative (IHC 0) in the PDECX models. Contrasting with this, three PDECX models showed HER2 $2+$ expression but the HER2 positivity was not detected in the corresponding patient
ESCC tissues. Multiple repeat experiments were performed to exclude technical error as a possible explanation for this result and the authors suggest tumor heterogeneity as a likely cause. Indeed, the location of the tissues used to establish the PDECX mouse models was different to that used to prepare formalin-fixed paraffin-embedded sections and perform genetic analysis. Another possibility may be clone enrichment or disappearance of human genetic aberrations within the mouse environment, however, this is speculation and is the subject of ongoing investigations. Taken together, these 
findings highlight the necessity for thorough characterization of patient-derived xenograft models before drug testing in preclinical drug discovery and development.

In our previously published study, ${ }^{30}$ Trastuzumab induced complete tumor regressions in PDECX mouse models with HER2 positivity (IHC $2+$ ), but was inefficacious in models harboring either PIK3CA mutation or combined HER2 IHC $2+$ positivity and PIK3CA mutation. In the present study, we extended these findings to demonstrate that PDECX models with HER2 negativity and wild-type PIK3CA were more sensitive to chemotherapy using 5-FU and cisplatin than PDECX models harboring genetic aberrations in HER2 or PIK3CA mutation.

In summary, a panel of PDECX mouse models, which included PIK3CA mutant and HER2-positive models, was established and characterized, thus mimicking the current clinical genetic setting of esophageal carcinoma. The sensitivity of HER2-negative ESCC models to chemotherapy supports stratification approaches in the treatment of esophageal carcinoma patients and warrants further investigation of the impact of PI3KCA on treatment response.

\section{ACKNOWLEDGMENTS}

We gratefully acknowledge the support of the patients and their families who agreed to contribute to this research program. This study received grant support for identification and establishment of Chinese patientderived esophageal carcinoma xenograft mouse model from Shanghai Science and Technology Committee, Shanghai Municipal Health Bureau Program (No. 11140902502).

\section{DISCLOSURE/CONFLICT OF INTEREST}

The authors declare no conflict of interest.

1. Montgomery EF, Boffetta P, Daigo Y, et al. WHO Classification of Tumors of the Oesophagus, 4th edn. IARC Press: Lyon, 2010.

2. Pennathur A, Gibson $\mathrm{MK}$, Jobe $\mathrm{BA}$, et al. Oesophageal carcinoma. Lancet 2013;381:400-412.

3. Enzinger PC, Mayer RJ. Esophageal cancer. N Engl J Med 2003;349: 2241-2252.

4. Kaifi JT, Gusani NJ, Jiang Y, et al. Multidisciplinary management of early and locally advanced esophageal cancer. J Clin Gastroenterol 2011:45:391-399.

5. Ilson DH. Oesophageal cancer: new developments in systemic therapy. Cancer Treat Rev 2003;29:525-532.

6. Shim HJ, Cho SH, Hwang JE, et al. Phase II study of docetaxel and cisplatin chemotherapy in 5-fluorouracil/cisplatin pretreated esophageal cancer. Am J Clin Oncol 2010;33:624-628.

7. Dipetrillo $\mathrm{T}$, Suntharalingam $\mathrm{M}, \mathrm{Ng} \mathrm{T}$, et al. Neoadjuvant paclitaxel poliglumex, cisplatin, and radiation for esophageal cancer: a phase 2 trial. Am J Clin Oncol 2012;35:64-67.

8. Jatoi A, Soori G, Foster NR, et al. Phase II study of preoperative pemetrexed, carboplatin, and radiation followed by surgery for locally advanced esophageal cancer and gastroesophageal junction tumors. J Thorac Oncol 2010;5:1994-1998.

9. van Hagen $\mathrm{P}$, Hulshof $\mathrm{MC}$, van Lanschot JJ, et al. Preoperative chemoradiotherapy for esophageal or junctional cancer. N Engl J Med 2012;366:2074-2084

10. Ruhstaller T, Widmer L, Schuller JC, et al. Multicenter phase II trial of preoperative induction chemotherapy followed by chemoradiation with docetaxel and cisplatin for locally advanced esophageal carcinoma (SAKK 75/02). Ann Oncol 2009;20:1522-1528.

11. Ruhstaller $T$, Pless $M$, Dietrich $D$, et al. Cetuximab in combination with chemoradiotherapy before surgery in patients with resectable, locally advanced esophageal carcinoma: a prospective, multicenter phase IB/ II Trial (SAKK 75/06). J Clin Oncol 2011;29:626-631.

12. Crosby T, Hurt CN, Falk $S$, et al. Chemoradiotherapy with or without cetuximab in patients with oesophageal cancer (SCOPE1): a multicentre, phase 2/3 randomised trial. Lancet Oncol 2013;14: 627-637.

13. Gibson MK, Malhotra U. Targeted therapy: an evolving concept in esophageal adenocarcinoma. Oncology (Williston Park) 2010;24: $11431146,1148$.

14. Gold PJ, Goldman B, Iqbal S, et al. Cetuximab as second-line therapy in patients with metastatic esophageal adenocarcinoma: a phase II Southwest Oncology Group Study (S0415). J Thorac Oncol 2010;5: 1472-1476.

15. Gros SJ, Kurschat N, Dohrmann T, et al. Effective therapeutic targeting of the overexpressed HER-2 receptor in a highly metastatic orthotopic model of esophageal carcinoma. Mol Cancer Ther 2010;9:2037-2045.

16. Mamede M, Abreu-E-Lima P, Oliva MR, et al. FDG-PET/CT tumor segmentation-derived indices of metabolic activity to assess response to neoadjuvant therapy and progression-free survival in esophageal cancer: correlation with histopathology results. Am J Clin Oncol 2007;30:377-388.

17. Safran H, Dipetrillo T, Akerman $P$, et al. Phase I/II study of trastuzumab, paclitaxel, cisplatin and radiation for locally advanced, HER2 overexpressing, esophageal adenocarcinoma. Int J Radiat Oncol Biol Phys 2007:67:405-409.

18. Safran H, DiPetrillo T, Nadeem A, et al. Trastuzumab, paclitaxel, cisplatin, and radiation for adenocarcinoma of the esophagus: a phase I study. Cancer Invest 2004;22:670-677.

19. Lee MS, Mamon HJ, Hong TS, et al. Preoperative cetuximab, irinotecan cisplatin, and radiation therapy for patients with locally advanced esophageal cancer. Oncologist 2013;18:281-287.

20. Morton CL, Houghton PJ. Establishment of human tumor xenografts in immunodeficient mice. Nat Protoc 2007;2:247-250.

21. Jin $\mathrm{K}$, Teng $\mathrm{L}$, Shen $\mathrm{Y}$, et al. Patient-derived human tumour tissue xenografts in immunodeficient mice: a systematic review. Clin Transl Oncol 2010;12:473-480.

22. Tentler JJ, Tan AC, Weekes CD, et al. Patient-derived tumour xenografts as models for oncology drug development. Nat Rev Clin Oncol 2012;9:338-350.

23. Garber K. From human to mouse and back: 'tumorgraft' models surge in popularity. J Natl Cancer Inst 2009;101:6-8.

24. Marangoni E, Vincent-Salomon A, Auger $\mathrm{N}$, et al. A new model of patient tumor-derived breast cancer xenografts for preclinical assays. Clin Cancer Res 2007:13:3989-3998.

25. Huynh H, Soo KC, Chow PK, et al. Xenografts of human hepatocellular carcinoma: a useful model for testing drugs. Clin Cancer Res 2006; $12: 4306-4314$

26. Huynh $\mathrm{H}$, Soo KC, Chow PK, et al. Targeted inhibition of the extracellular signal-regulated kinase kinase pathway with AZD6244 (ARRY-142886) in the treatment of hepatocellular carcinoma. Mol Cancer Ther 2007;6:138-146.

27. Huynh $\mathrm{H}$, Lee JW, Chow PK, et al. Sorafenib induces growth suppression in mouse models of gastrointestinal stromal tumor. Mol Cancer Ther 2009;8:152-159.

28. Fichtner I, Rolff J, Soong R, et al. Establishment of patient-derived nonsmall cell lung cancer xenografts as models for the identification of predictive biomarkers. Clin Cancer Res 2008;14:6456-6468.

29. Daniel VC, Marchionni L, Hierman JS, et al. A primary xenograft model of small-cell lung cancer reveals irreversible changes in gene expression imposed by culture in vitro. Cancer Res 2009;69: 3364-3373.

30. Wu X, Zhang J, Zhen R, et al. Trastuzumab anti-tumor efficacy in patient-derived esophageal squamous cell carcinoma xenograft (PDECX) mouse models. J Transl Med 2012;10:180.

31. Hofmann M, Stoss O, Shi D, et al. Assessment of a HER2 scoring system for gastric cancer: results from a validation study. Histopathology 2008:52:797-805.

32. Kurosumi M. Recent trends of HER-2 testing and trastuzumab therapy for breast cancer. Breast Cancer 2009;16:284-287.

33. Sato-Kuwabara $\mathrm{Y}$, Neves $\mathrm{Jl}$, Fregnani $\mathrm{JH}$, et al. Evaluation of gene amplification and protein expression of HER-2/neu in esophageal squamous cell carcinoma using Fluorescence in situ Hybridization (FISH) and immunohistochemistry. Bmc Cancer 2009;9:6. 
34. Hou J, Jiang DX, Zhang JC, et al. Frequency, characterization, and prognostic analysis of PIK3CA gene mutations in Chinese esophageal squamous cell carcinoma. Hum Pathol 2014;45:352-358.

35. Wu S, Zhu Z, He J, et al. A novel mutant-enriched liquidchip technology for the qualitative detection of somatic mutations in KRAS gene from both serum and tissue samples. Clin Chem Lab Med 2010; 48:1103-1106.

36. Sausville EA, Burger AM. Contributions of human tumor xenografts to anticancer drug development. Cancer Res 2006;66:3351-3354, discussion 3354.

37. Opitz OG, Harada H, Suliman $Y$, et al. A mouse model of human oralesophageal cancer. J Clin Invest 2002;110:761-769.

38. Twarock S, Freudenberger T, Poscher E, et al. Inhibition of oesophageal squamous cell carcinoma progression by in vivo targeting of hyaluronan synthesis. Mol Cancer 2011;10:30.

39. Lange T, Nentwich MF, Luth M, et al. Trastuzumab has anti-metastatic and anti-angiogenic activity in a spontaneous metastasis xenograft model of esophageal adenocarcinoma. Cancer Lett 2011;308:54-61.

40. Ali R, Barnes I, Kan SW, et al. Cancer incidence in British Indians and British whites in Leicester, 2001-2006. Br J Cancer 2010;103:143-148.

41. Deng YJ, Rong TH, Zhou J, et al. [Establishment of a human esophageal carcinoma transplantation model with MUC1 high expression in nude mice]. Ai Zheng 2007;26:693-697.

42. Ohara T, Takaoka M, Sakurama K, et al. The establishment of a new mouse model with orthotopic esophageal cancer showing the esophageal stricture. Cancer Lett 2010;293:207-212.

43. Fiebig $\mathrm{HH}$, Maier A, Burger AM. Clonogenic assay with established human tumour xenografts: correlation of in vitro to in vivo activity as a basis for anticancer drug discovery. Eur J Cancer 2004;40:802-820.

44. Kitamura M, Suda M, Nishihira T, et al. Heterotransplantation of human esophageal carcinoma to nude mice. Tohoku J Exp Med 1981;135: 259-264.
45. Dodbiba L, Teichman J, Fleet A, et al. Primary esophageal and gastroesophageal junction cancer xenograft models: clinicopathological features and engraftment. Lab Invest 2013;93:397-407.

46. Fichtner I, Slisow W, Gill J, et al. Anticancer drug response and expression of molecular markers in early-passage xenotransplanted colon carcinomas. Eur J Cancer 2004;40:298-307.

47. Borgmann A, Baldy C, von Stackelberg A, et al. Childhood all blasts retain phenotypic and genotypic characteristics upon long-term serial passage in NOD/SCID mice. Pediatr Hematol Oncol 2000;17: 635-650.

48. Park B, Jeong BC, Choi YL, et al. Development and characterization of a bladder cancer xenograft model using patient-derived tumor tissue. Cancer Sci 2013;104:631-638.

49. Fichtner I, Becker $\mathrm{M}$, Zeisig $\mathrm{R}$, et al. In vivo models for endocrinedependent breast carcinomas: special considerations of clinical relevance. Eur J Cancer 2004;40:845-851.

50. Boland PM, Burtness B. Esophageal carcinoma: are modern targeted therapies shaking the rock? Curr Opin Oncol 2013;25:417-424.

51. Mimura K, Kono K, Hanawa $M$, et al. Frequencies of HER-2/neu expression and gene amplification in patients with oesophageal squamous cell carcinoma. Br J Cancer 2005;92:1253-1260.

52. Langer CJ, Stephenson $\mathrm{P}$, Thor A, et al. Trastuzumab in the treatment of advanced non-small-cell lung cancer: is there a role? Focus on Eastern Cooperative Oncology Group study 2598. J Clin Oncol 2004:22:1180-1187.

53. Gravalos C, Jimeno A. HER2 in gastric cancer: a new prognostic factor and a novel therapeutic target. Ann Oncol 2008;19:1523-1529.

54. Bang $Y J$, Van Cutsem E, Feyereislova A, et al. Trastuzumab in combination with chemotherapy versus chemotherapy alone for treatment of HER2-positive advanced gastric or gastro-oesophageal junction cancer (ToGA): a phase 3, open-label, randomised controlled trial. Lancet 2010;376:687-697. 